\title{
Ordnance Survey Digital Data in UK Legal Deposit Libraries
}

\author{
by CHRISTOPHER FLEET
}

\section{INTRODUCTION}

In common with many other national mapping organisations, the British Ordnance Survey (OS) has increasingly shifted away from paper publications to digital methods of production over the last twenty years. Indeed, to an extent the OS have been pioneers in this process, investigating early digital techniques in the 1960s, and converting the whole of its basic scale mapping into a digital form by 1995. Also, in contrast to many other national mapping agencies, this basic scale mapping is very detailed, with urban areas mapped at $1: 1,250$ scale, lowland rural areas at 1:2,500, and all other areas mapped at $1: 10,000$. For anyone interested in Great Britain's landscape over the last two hundred years, this mapping is the essential detailed archive of national topography, used by a wide range of private and public organisations and individuals. For the six legal deposit libraries in the United Kingdom and Republic of Ireland ${ }^{1}$ this mapping is the most frequently consulted body of material, and their archives of OS maps date back to the inception of the OS, with comprehensive legal deposit established from 1911. However, in recent years the mapping has shifted away from paper publication. From the late 1970s the main new media for the archiving of large-scale mapping were Survey Information on Microfilm (SIM) and the proportion of these has grown so that today all 1:2,500 and 1:1,250 mapping is received on $\mathrm{SIM}^{2}$. But with the confirmation by OS that SIM could no longer be produced after March 1999, the search commenced for a totally digital means of receiving OS mapping.

\section{BACKGROUND}

Many of the broader issues concerning this transition were aired at the Future History of our Landscape Seminar ${ }^{3}$, held at the Royal Society in London in October 1992, and have informed subsequent decisions made on the subject. 
Ordnance Survey Digital Data in UK Legal Deposit Libraries

For example, the need to move from traditional paper mapping and archive new digital media, the need for the Copyright Libraries and the Public Record Office to agree new arrangements with the OS, and the importance of maintaining access to digital topographic information for all user groups in the future were acknowledged by all. In addition, many of the problems discussed at that meeting have become more pertinent, in particular, the government pressure on OS to recover costs and restrict free access to its data, the problem of data standardisation, durability of media and technological change, and the extra costs for libraries and archives in handling digital data. These will be discussed in more detail below.

For the legal deposit libraries, the subject was actively discussed with OS at the half-yearly meetings of BRICMICS (British and Irish Committee for Map Information and Catalogue Systems), culminating in an agreement reached in April 1997. Essentially this confirmed that from 1999 the OS would supply its National Topographic Database as an annual snapshot to each of the six legal deposit libraries. The libraries have been particularly grateful to OS in making this offer, as there is still no legal requirement to deposit digital data in the United Kingdom, and the true market cost of the data would have been way beyond the budgets of the Copyright Libraries. At this time BRICMICS set up a Digital Data Working Group with representatives from OS, the PRO, the British Library, the Bodleian Library, Cambridge University Library, and the National Library of Scotland to evaluate the main computing hardware, software and archival options. The more technical issues have been investigated by the British Library Information Systems Department in close collaboration with members of the Group, and they have prepared a detailed Feasibility Study, and financed the customisation of the trial viewing software.

\section{DATA FORMAT}

Following some discussion over the definition of the National Topographic Database, agreement was reached for the OS to supply its Land-Line data product to the legal deposit libraries. This is the main vector point-and-line database of large-scale topography, at the nominal scales of 1:10,000, 1:2,500, and 1:1,250 depending on the geographic area. Unfortunately, this database does not include contours, and as the OS was in a process of re-engineering its contours database, it recommended that the supply of this information be deferred for future agreement. For many upland areas, the importance of contours as a vital element of topography must be stressed, and we will certainly need to add contours in the future to make these and other areas more comprehensible as maps on the screen. 
The Land-Line data is to be supplied in the National Transfer Format (NTF), an independent data format (BS 7567) maintained by the Association for Geographic Information, and the main format for all OS data. It is structured into tiles that correspond to the areas covered by traditional paper maps, and therefore monitoring change through time by tiles is possible. The format has the advantages of being non-proprietary, well-documented, with a comprehensive and consistent structure, and readable by any ASCII text editor. However, in common with many geographic data formats, it requires special software to view it, and is subject to periodic revision, having a number of earlier versions. Indeed, during 1997 and 1998 OS has been re-engineering its own internal database ${ }^{4}$, and this may well alter the data supplied to the Copyright Libraries.

\section{SOFTWARE}

\subsection{OSView}

Initially, the OS recommended their free and simple viewer, OSView, which was practically evaluated with some test mapping data files in the British Library and National Library of Scotland ${ }^{5}$. Although clear and simple to operate and allowing extensive customisation of features for display, the software had a number of important limitations. In particular, it could only display one data tile at a time, and therefore areas covering more than one tile could not be simultaneously displayed. It also provided no easy means of searching for tiles, requiring the user to locate and open the required tile by the tile name alone. Finally, in that OS felt unable to support or modify the software at that time, it would have been impossible to customise it to meet the libraries' needs and respond to changing requirements in future years. The limitations of OSView therefore forced the Group to explore other products.

\subsection{Readers' Requirements}

In order to systematically compare these alternatives, a list of readers' requirements was drafted, covering the main functional areas such as search facilities, viewing, metadata, printing, and archiving. These were prioritised by the Group into those that were mandatory for the project, and those that could be left for future consideration. A shortlist of potential GIS software packages was then prepared and evaluated using the requirements, and the advice of a number of experts in digital mapping was sought, including the Digimap (see paper Nick Millea elsewhere in this issue) team at Edinburgh 
Ordnance Survey Digital Data in UK Legal Deposit Libraries

University. Following this process the decision was taken in February 1998 to customise the desktop mapping package MapInfo, tailoring its functions for the libraries' requirements.

\subsection{MapInfo}

The decision to use MapInfo was based particularly on the ease with which it could be customised using MapBASIC, the widespread popularity and user base of MapInfo, and its basis and performance within a Windows PC environment. The customisation was undertaken by a company called Dotted Eyes, based in Birmingham, United Kingdom, who have developed software for a number of geographic data products, including a converter for OS NTF data. Both MapBASIC and Delphi were used to create a viewer for the data, and the source code was made available as part of the contract, so that further customisation by the libraries will be possible. On the one hand the customisation eliminated certain functions within MapInfo deemed irrelevant for library users, and on the other added extra features for selecting tiles and displaying data. Ordnance Survey specified that many standard GIS functions that MapInfo could perform should be eliminated, thereby aiming to re-create the traditional paper map use practices in the digital environment. The customisation was completed by July 1998, and the software was tested and evaluated in the British Library and National Library of Scotland from August 1998.

Figure 1 shows the standard screen user interface. Searching for an area is possible by National Grid Reference or using a basic gazetteer (a zoomable display of smaller-scale mapping for locating an area unfortunately proved too costly to purchase, though it could be added in future). Having located the required area, buttons on each side of the map display allow all basic functions to be performed, such as zooming in and out, moving across the map display, viewing metadata information, etc. A small overview window for locating the main display was felt to be desirable, and the cursor position is constantly given in National Grid co-ordinates, along with the screen scale. It is also possible to display the same area at different points in time by a dual-window arrangement (Figure 2). However, all these features may be subject to alteration in response to user feedback. 


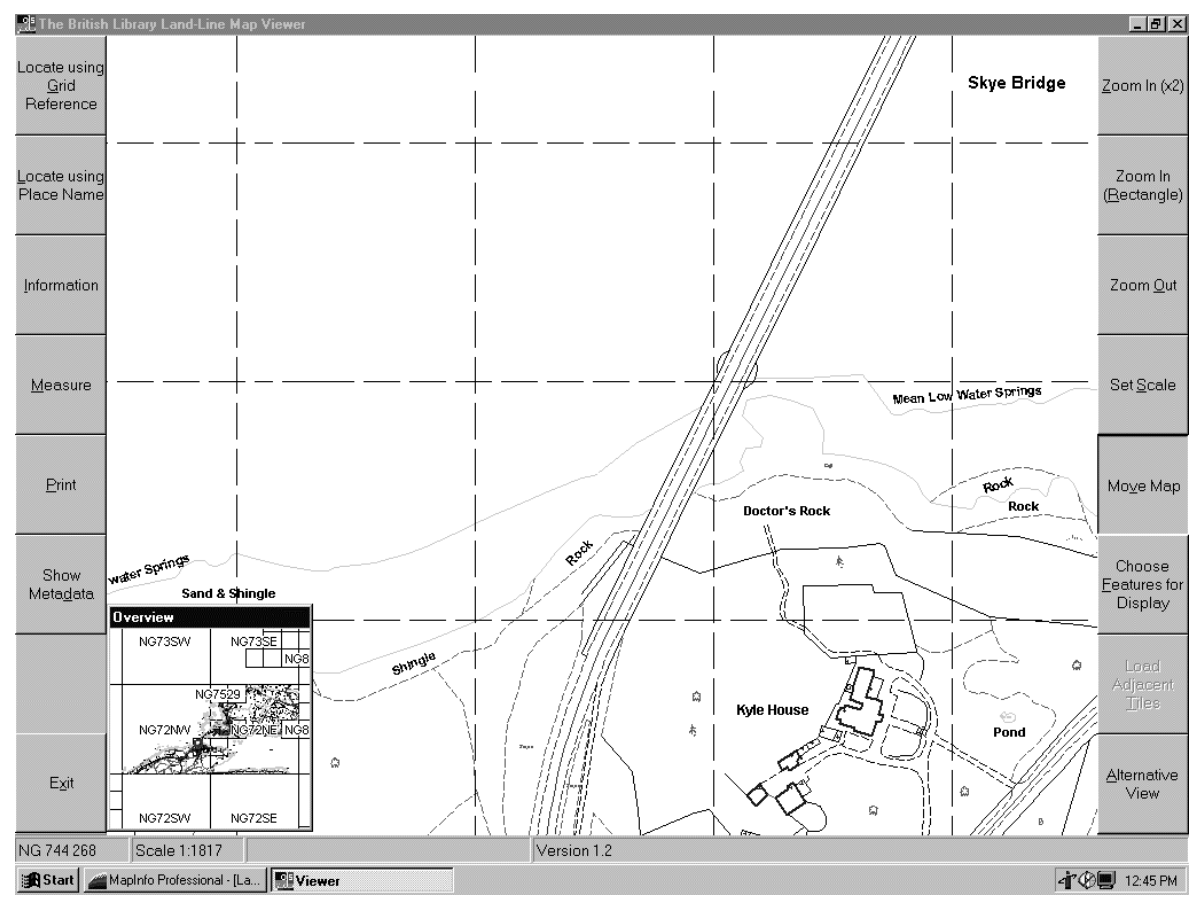

Figure 1. The standard screen interface for the MapInfo Viewer, showing Land-Line data for part of the Skye Bridge in Scotland

\section{Metadata and Recording Change}

To any user of OS paper mapping, the date of survey or revision is crucial in dating the map. This is no less true in a digital age, although for a continuously changing database, most tiles display features surveyed at a number of different points in time. A fundamental question for the data user and archivist is therefore how can the digital map being displayed be dated? Within the NTF there are two fields which record change for every tile: a Latest Amended Date (the date when a tile was last amended) and a House Unit Count (a continually growing number of house units of change since the last paper edition). These fields are therefore recorded into a catalogue database as metadata when the NTF files are converted into MIF, and are used directly by the viewing software in selecting and displaying maps. Although individual map features cannot be date stamped, some approximate dating of the map display is possible using these fields. 


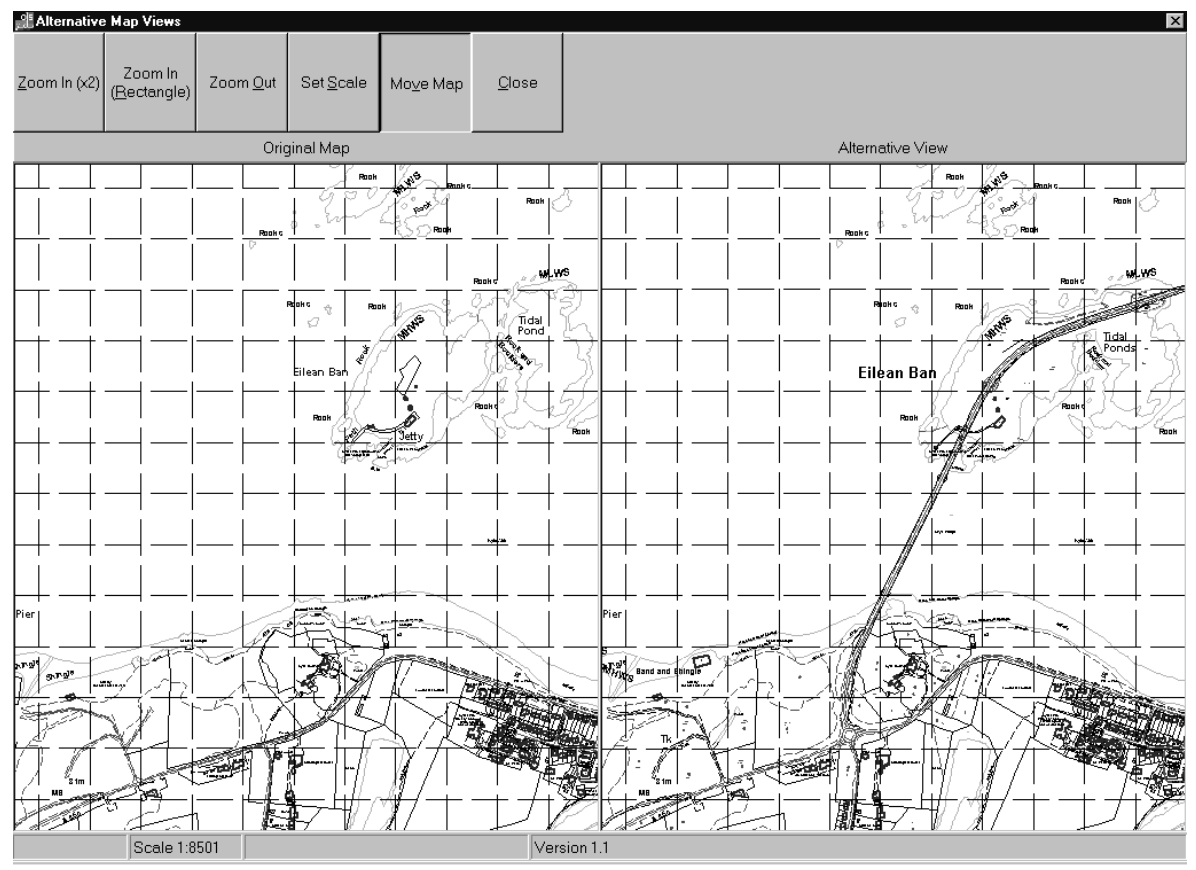

Figure. 2: Dual-window screen display in the MapInfo Viewer for comparing different dates for the same area - in this example before and after the construction of the Skye Bridge.

A second archival problem of continuously updating databases, acknowledged by the Future History of the Landscape Seminar, was the question of how change itself needs to be archived. Essentially there are two main archival solutions: a record of change approach, receiving data after a certain amount of change has occurred, versus a snapshot approach, taking all data at a particular point in time. Historically, on paper mapping, the former has always been the approach, with new editions of paper mapping only being published after a certain number of house units of change. Thus for a $1 \mathrm{x} 1 \mathrm{~km}$ 1:2,500 paper map, a new edition was published after 300 house units of change, reduced to 50 units on SIM and 20 for Superplan microfilm cards. (Microfilm cards were therefore generated more regularly than paper mapping and their archiving of change was more regular). For future digital data received by the legal deposit libraries from Ordnance Survey, an annual snapshot is the preferred method, in part because this is already performed for OS security backup purposes, and is easiest to manage and distribute ${ }^{6}$. On average, each tile has only 4 house units of change per annum, and therefore, in theory at least, future digital landscape change will be recorded more 
regularly. The snapshot approach also requires the NTF translation software to only archive tiles that have changed in second and subsequent years, so that tiles that have not changed (and are therefore identical in successive snapshots) are recorded in the libraries' database only once.

\section{SECURITY AND ACCESS TO THE DATA}

The importance of storing the OS data securely has been paramount in the Group's decisions, and the chosen model of data delivery will ensure that there is no danger of data leakage. The plan is for OS to supply the data using their preferred digital media (currently CD-ROM but this may change in future with technology) to standalone workstations in the legal deposit libraries. There are no plans for online access or exporting of data beyond the libraries, and therefore controlling the use of the data will be much easier. Although discussions were still underway at the time of writing, the Copyright Libraries are hoping to continue access arrangements to digital data that have been allowed in the past for paper mapping and SIM.

\section{HARDWARE}

The Land-Line database is estimated to be 38 gigabytes in size, and can be supplied on $57 \mathrm{CD}-\mathrm{ROMs}$, although the size and media may well change in future years. After the first year, only files that have changed will be archived, and this is estimated to be around 23 gigabytes every year. Although offline storage on CD-ROM, tape or other media is a possibility, the need for relatively quick access to data, and the falling costs of hard disk memory will further tip the balance in favour of storing all the data on a single computer workstation.

Whilst it is true that the costs of this hardware are a substantial addition to the Libraries' budgets, the shift to new media will allow significant savings to be made elsewhere. Over two years, the money spent on hardware will actually be less than the amount paid to OS for mounting the SIM copycards, and there will be significant savings on staff time in not needing to file several thousand SIM every year. In addition, the costs of computer hardware will certainly continue to fall in future, and the time taken to transfer and convert data will reduce. The overall financial balance sheet is therefore quite complex, and although the initial costs of digital archiving are higher, the long term costs may be lower. 


\section{Some advantages and disadvantages of OS digital data in UK legal deposit libraries}

\section{Advantages}

- Easier means of searching via placename gazetteer

- Easier display of "seamless" data

- Zoomable display of data at various scales

- Colour coding of features to aid interpretation

- More regular archiving of change with annual snapshot

- Less filing of SIM and paper maps for staff

\section{Disadvantages}

- High specification machinery with rapidly growing storage requirements

- Programming of software to view and maintain data

- Data conversion and migration in future years

- Energy consumption of computer machinery

- New staff responsibilities, tasks and training requirements

Table 1.

\section{CONCLUSION}

Technology should be merely a means of allowing continued access to mapping, and therefore to conclude it is important to realise how this new digital technology has both positive and negative aspects (Table 1). From a user perspective, there are several advantages in searching for digital maps with the software and display (using colour) and with more regular digital archiving. The disadvantages are the additional resources required to manage, store, and migrate the data, the need to program software to view it, and the shift in staff responsibilities. Whether the digital data is easier to use than paper maps depends very much on the user and their priorities, as although the viewing software is relatively simple, we must not forget that the paper medium will continue to have its advantages over digital data for many years, and is still (arguably) the preferred medium for the majority of our current map users. 


\section{REFERENCES}

1 The Bodleian Library, Oxford, the British Library, Cambridge University Library, National Library of Scotland, National Library of Wales, and Trinity College Dublin.

2 NLS estimate that they hold over 80,000 SIM for the whole UK and currently receive on average about 8,000 new SIM every year.

3 Christopher Board and Peter Lawrence (eds.) Recording our changing landscape: the proceedings of the Seminar on the Future History of our Landscape held at The Royal Society on 16th October 1992. London: The Royal Society and The British Academy in association with The British Cartographic Society, 1994.

4 Ordnance Survey Enhancing the National Topographic Database (NTD). Consultation Paper 2/1997, and Ordnance Survey Enhancing the National Topographic Database (NTD). Information Paper 1/1998.

5 This software has since been developed and can be freely downloaded from the Ordnance Survey website (<http://www.ordsvy.gov.uk/samples/Osview/ index.htm $>$ ) Although it can now view more than one tile at a time, its other limitations still hold true.

6 An annual snapshot is also the preferred method for archiving OS data for the PRO. The OS is now a place of deposit under the Public Records Act (1958, 1967) for the electronic copy of the National Topographic Database. Ordnance Survey Historical maps and map data. Consultation Paper 4/1997, and Ordnance Survey Historical maps and map data. Information Paper 3/1998.

Christopher Fleet

Map Library

National Library of Scotland

33 Salisbury Place

Edinburgh EH9 1SL, United Kingdom

c.fleet@nls.uk 